Invited Review presented at the GeV-TeV Gamma-ray Astrophysics Workshop "Towards a Major Atmospheric Cerenkov Detector VI" Snowbird, Utah - August 13-16, 1999.

\title{
The AGILE gamma-ray astronomy mission
}

\author{
S. Mereghetti ${ }^{1}$, G. Barbiellini ${ }^{2}$, G. Budini ${ }^{2}$, P. Caraveo ${ }^{1}$, E. Costa ${ }^{3}$,

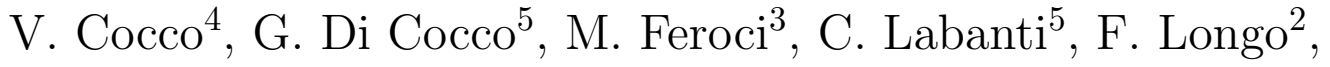 \\ E. Morelli ${ }^{5}$, A. Morselli ${ }^{4}$, A. Pellizzoni $^{6}$, F. Perotti ${ }^{1}$, P. Picozza $^{4}$, \\ M. Prest ${ }^{2}$, P. Soffitta ${ }^{3}$, L. Soli $^{1}$, M. Tavani ${ }^{1}$, E. Vallazza $^{2}$, \\ S. Vercellone ${ }^{1}$ \\ ${ }^{1}$ Istituto di Fisica Cosmica G.Occhialini - CNR, Milano, Italy \\ ${ }^{2}$ Università di Trieste and INFN, Trieste, Italy \\ ${ }^{3}$ Istituto di Astrofisica Spaziale - CNR, Roma, Italy \\ ${ }^{4}$ Università "Tor Vergata" and INFN, Roma, Italy \\ ${ }^{5}$ Istituto TESRE - CNR, Bologna, Italy \\ ${ }^{6}$ Agenzia Spaziale Italiana
}

\begin{abstract}
We describe the AGILE gamma-ray astronomy satellite which has recently been selected as the first Small Scientific Mission of the Italian Space Agency. With a launch in 2002, AGILE will provide a unique tool for high-energy astrophysics in the 30 $\mathrm{MeV}$ - $50 \mathrm{GeV}$ range before GLAST. Despite the much smaller weight and dimensions, the scientific performances of AGILE are comparable to those of EGRET.
\end{abstract}

\section{INTRODUCTION}

The AGILE satellite was proposed in June 1997 to the Program for Small Scientific Missions of the Italian Space Agency (ASI). AGILE (Astro-rivelatore Gamma a Immagini LEggero) is a mission devoted to gamma-ray (30 MeV-50 GeV) astrophysics during the years 2002-2005 After the initial ASI selection in December 1997, a Phase A study was carried out during 1998. AGILE was finally selected by ASI in June 1999 as the first Small Scientific Mission to be launched and is currently in the Phase B. The launch is foreseen in early 2002.

The AGILE scientific payload is based on the state-of-the-art and reliably developed technology of solid state silicon detectors [1-3]. The instrument is very light $(\sim 60 \mathrm{~kg})$ and effective in detecting and monitoring gamma-ray sources $(30 \mathrm{MeV}-$ $50 \mathrm{GeV}$ ) within a large field of view. The instrument is designed to achieve an 
optimal angular resolution (source location accuracy $\sim 5^{\prime}-20^{\prime}$ for intense sources), a very large field of view ( $\gtrsim 2 \mathrm{sr}$ ), and a sensitivity comparable to that of EGRET for on-axis (and substantially better for off-axis) point sources. AGILE will also carry an imaging hard X-ray detector to simultaneously monitor in the $10-40 \mathrm{keV}$ range the sources observed in the central part of the gamma-ray field of view.

Despite its simplicity and moderate cost, AGILE is ideal to perform a large number of tasks [4]: monitoring active galactic nuclei, detecting gamma-ray bursts with high efficiency, mapping the diffuse Galactic and extragalactic emission, studying pulsed gamma-ray emission from radiopulsars, monitoring the many unidentified sources and contributing to their unveiling, detecting energetic solar flares . Today, it is clear that successful investigations of gamma-ray sources rely on coordinated space and ground-based observations. The AGILE scientific program will be focussed on a prompt response to gamma-ray transients and alert for follow-up multiwavelength observations.

\section{INSTRUMENT OVERVIEW}

The AGILE scientific payload is made of three main detectors: a silicon/tungsten Tracker, a cesium iodide Mini-calorimeter, and a coded mask hard X-ray Imaging Detector. These three elements are integrated in a single structure, covered on five sides (top and lateral sides) with an Anticoincidence for charged particles rejection.

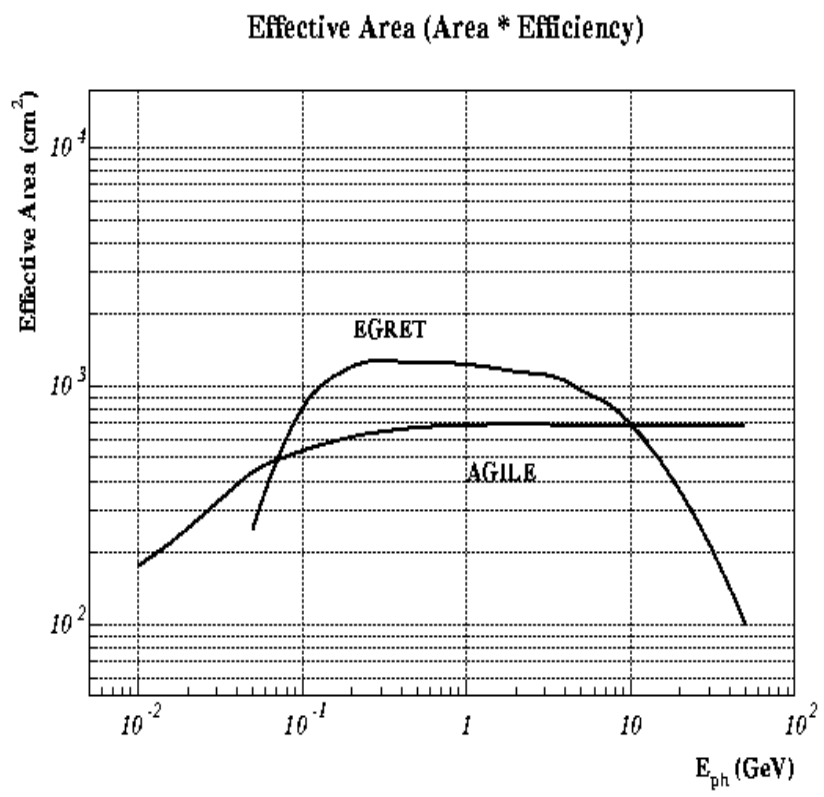

FIGURE 1. Effective area of the AGILE gamma-ray detector. 
The Tracker, consisting of 14 planes of silicon strip detectors, will provide the unambiguous identification of incident gamma-rays by recording the characteristic track signature of the $\mathrm{e}^{-}-\mathrm{e}^{+}$that result from pair creation from the incident photons in thin layers converting material. Each plane of the Tracker (with the exception of the two at the bottom) is made of three layers: a $245 \mu \mathrm{m}$ thick photon pair converter in tungsten $\left(0.07 X_{0}\right)$ is followed by two planes of silicon strip detectors (thickness $410 \mu \mathrm{m}$ ) with the strips arranged in orthogonal directions to provide the plane coordinates of the particle tracks. The tungsten layer is absent in the last two planes, since the readout trigger requires a signal in at least three consecutive silicon planes to be activated. The Tracker has an on-axis total radiation length larger than $0.84 X_{0}$. The resulting on-axis effective area is shown in Figure 1. The distance between planes has been fixed to $1.6 \mathrm{~cm}$ on the basis of extensive optimizations by Montecarlo simulations.

The fundamental unit for the silicon planes is a module of area $9.5 \times 9.5 \mathrm{~cm}^{2}$, and pitch (distance between strips) equal to $121 \mu \mathrm{m}$. Each silicon plane consists of $4 \times 4$ modules. The strips will be read out with a pitch of $242 \mu \mathrm{m}$, i.e. with a floating strip every two strips, yielding a total of $\sim 43,000$ channels for the whole Tracker. If a particle crosses a floating strip, image charges are induced on the two adjacent read-out strips through capacitive coupling. In this way, more than one strip has signal, thus enabling to use an interpolation algorithm to improve the spatial resolution. Special algorithms applied off-line to telemetered data will allow an optimal reconstruction of the photon incidence angle.

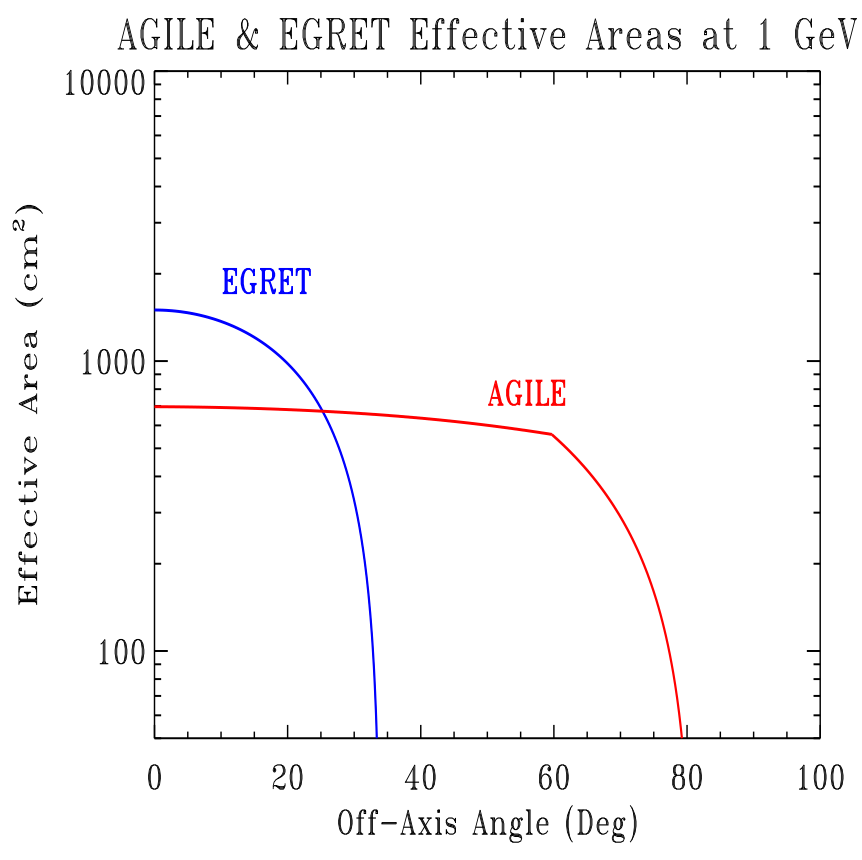

FIGURE 2. Comparison of the AGILE and EGRET effective areas for off-axis sources. 
The Mini-calorimeter, consisting of $1.5 X_{0}$ of Cesium Iodide (CsI), will allow to determine the energy of the incident photons imaged by the Tracker. In addition, the Mini-calorimeter will also be used to study gamma-ray bursts and other transient phenomena in the $\sim 1-500 \mathrm{MeV}$ energy range. The Mini-calorimeter is located below the Tracker and consists of two planes of $\mathrm{CsI}(\mathrm{Tl})$ bars. Each bar has dimensions of $2.3 \times 1.5 \times 40 \mathrm{~cm}^{3}$. The two planes have the bars arranged in orthogonal directions to provide the $\mathrm{X}$ and $\mathrm{Y}$ location of the showers. The scintillation light from each bar is collected by two photodiodes placed at both ends. We note that the problem of particle backscattering for this configuration is much less severe than in the case of EGRET, thus allowing a relatively efficient detection of photons up to $10 \mathrm{GeV}$.

The hard X-ray detector (Super-AGILE) is based on the $9.5 \mathrm{x} 9.5 \mathrm{~cm}^{2}$ silicon tiles that are used for the Tracker planes. These will be placed on the top of the Tracker (above the first tungsten layer) to form an additional detection plane sensitive in the 10-40 keV range and used in conjunction with a coded mask at a distance of about $10 \mathrm{~cm}$ (below the top Anticoincidence). Since the silicon microstrips provide pixels only along one-dimension, Super-AGILE will consist of four equal modules arranged in two pairs, giving monodimensional sky images along two orthogonal directions. The mask will be supported by an ultra-light structure in carbon fiber that will also serve as a collimator to reduce the Super-AGILE field of view. This is needed in order to limit the Super-AGILE background, which is dominated by the cosmic diffuse radiation.

The Anticoincidence system, aimed at both charged particle background rejection and preliminary direction reconstruction for triggered photon events, completely surrounds the top and lateral sides of the Super-AGILE, Tracker and Mini-

TABLE 1. AGILE Scientific Performances

\begin{tabular}{|c|c|c|}
\hline \multicolumn{3}{|c|}{ Gamma-ray Detector } \\
\hline Energy Range & $30 \mathrm{MeV}-50 \mathrm{GeV}$ & \\
\hline Field of view & $2 \mathrm{sr}$ & \\
\hline Sensitivity at $100 \mathrm{MeV}$ & $6 \times 10^{-9} \mathrm{ph} \mathrm{cm}^{-2} \mathrm{~s}^{-1} \mathrm{MeV}^{-1}$ & $\left(5 \sigma\right.$ in $\left.10^{6} \mathrm{~s}\right)$ \\
\hline Sensitivity at $1 \mathrm{GeV}$ & $4 \times 10^{-11} \mathrm{ph} \mathrm{cm}^{-2} \mathrm{~s}^{-1} \mathrm{MeV}^{-1}$ & $\left(5 \sigma\right.$ in $\left.10^{6} \mathrm{~s}\right)$ \\
\hline Angular Resolution at $1 \mathrm{GeV}$ & $36 \operatorname{arcmin}$ & ( $68 \%$ containment radius) \\
\hline Source Location Accuracy & $\sim 30$ arcmin & for a source with $\mathrm{S} / \mathrm{N} \sim 10$ \\
\hline Energy Resolution & $\Delta \mathrm{E} / \mathrm{E} \sim 1$ & at $300 \mathrm{MeV}$ \\
\hline Timing Accuracy & $25 \mu \mathrm{s}$ & \\
\hline \multicolumn{3}{|c|}{ Hard X-ray Detector } \\
\hline Energy Range & $10-40 \mathrm{keV}$ & \\
\hline Field of view & $60^{\circ} \times 60^{\circ}$ & Full Width at Zero Sensitivity \\
\hline Sensitivity & $\sim 10$ milliCrabs & $(5 \sigma$ in 1 day $)$ \\
\hline Angular Resolution & $10 \operatorname{arcmin}$ & \\
\hline Source Location Accuracy & $\sim 2-3$ arcmin & for a source with $\mathrm{S} / \mathrm{N} \sim 10$ \\
\hline Energy Resolution & $\Delta \mathrm{E}<4 \mathrm{keV}$ & \\
\hline Timing Accuracy & $25 \mu \mathrm{s}$ & \\
\hline
\end{tabular}


calorimeter. The top panel is a single slab of plastic scintillator with thickness 0.5

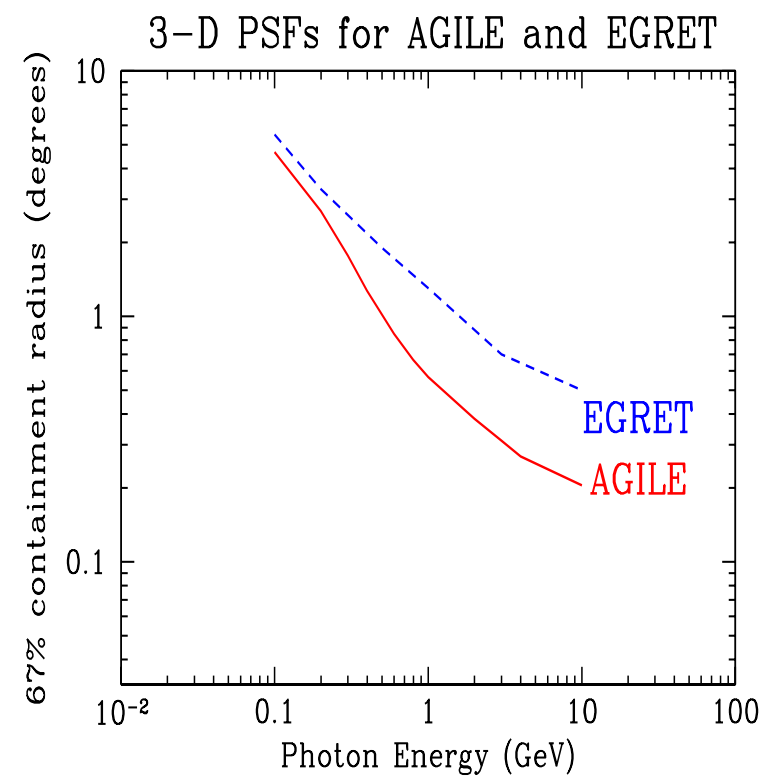

FIGURE 3. AGILE angular resolution.

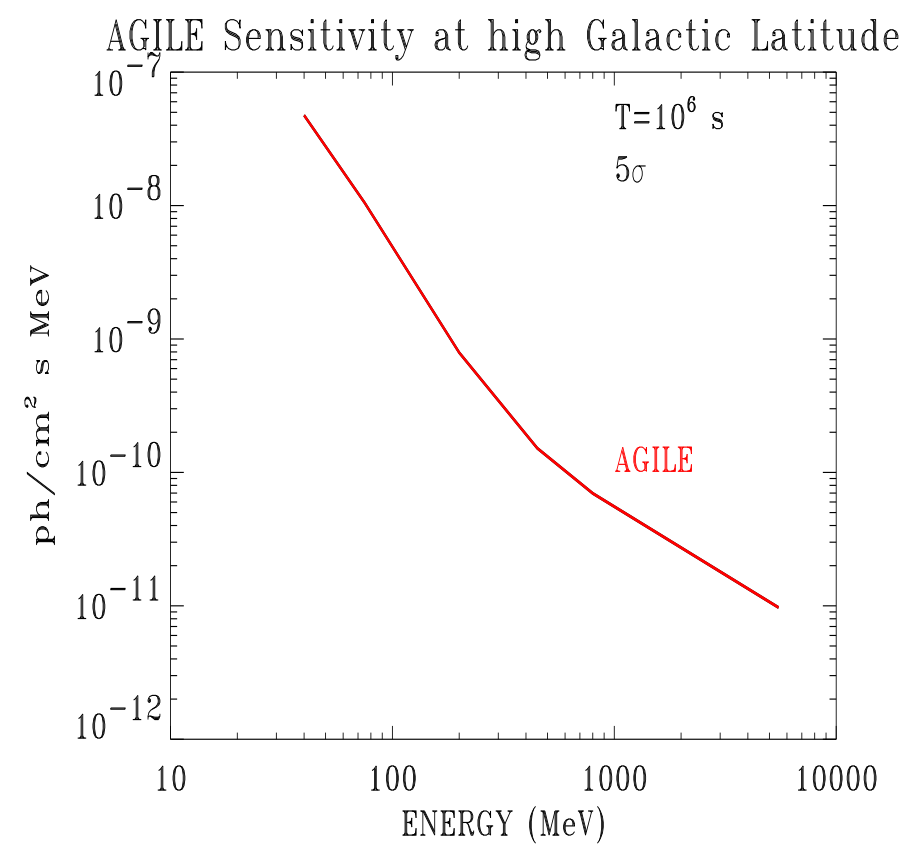

FIGURE 4. Expected sensitivity for the AGILE gamma-ray instrument. 
$\mathrm{cm}$. The scintillation light is collected by optical fibers glued on the four sides and directed to four photomultipier tubes at the corners. Each lateral face is segmented with three partially overlapping plastic scintillator layers $(0.5 \mathrm{~cm}$ thick $)$ connected with photomultipliers placed at the bottom.

\section{PERFORMANCES}

The expected scientific performances of AGILE are summarized in Table 1. One of the main characteristics of the AGILE gamma-ray detector is the very large field of view. As shown in Figure 2 the AGILE effective area remains almost constant for large off-axis angles. This will allow to simultaneously monitor a large number of sources in a single pointing and will also result, at the end of the mission, in a large exposure factor for each region of the sky [5].

The great sky exposure factor, coupled with the good angular resolution (see Figure 3), will allow a detailed study of the diffuse Galactic and Extra-Galactic emission and to better locate the unidentified EGRET sources.

Another important characteristic of the AGILE Tracker is the small dead time. This will be crucial in the study of the high energy emission of gamma-ray bursts, which are expected in the field of view at a rate of $\sim 5-10$ per year, based on the EGRET results.

The extension in the hard $\mathrm{X}$-ray range of a gamma-ray mission, made possible by the Super-AGILE detector, is an innovative concept that will allow the study of correlated variability for sources of different classes, ranging from active galactic nuclei and blazars to unidentified galactic transients.

\section{THE MISSION}

The AGILE instrument will be carried in an equatorial (inclination $<5^{\circ}$ ), circular orbit (altitude $\sim 550 \mathrm{~km}$ ), by a spacecraft of the MITA class, which is currently being developed by Gavazzi Space as prime contractor. The total mass will be of the order of 180-200 kg, including $\sim 60 \mathrm{~kg}$ of scientific payload.

The satellite will point with a 3 -axis attitude stabilization with an accuracy of the order of $1^{\circ}$. An attitude reconstruction at the arcmin level will be obtained a posteriori, by means of star sensors. A typical AGILE pointing will last 2-3 weeks. To maximise the observing efficiency, we are investigating the possibility of slewing to secondary pointing directions during the fraction of the orbit in which the primary target direction is occulted by the Earth. AGILE will also have the possibility to quickly repoint (within 1 day) in order to react to interesting targets of opportunity.

The operations center will have the primary responsibility of satellite operations and communications using the ASI ground base at Malindi (Kenya). The equatorial orbit will allow a single contact per orbit at a downlink rate of $500 \mathrm{kbit} \mathrm{s}^{-1}$. 
An AGILE data center will be devoted to the monitoring of the instrument scientific performance, to the quick look analysis, and to the processing, distribution and archival of scientific data products and the associated calibration data.

\section{CONCLUSIONS}

AGILE will provide crucial information complementary to the many lower energy detectors that will be operational during the first decade of the new Millenium (INTEGRAL, XMM, AXAF, ASTRO-E, SPECTRUM-X, and others). No other gamma-ray mission dedicated to the energy band above $\sim 30 \mathrm{MeV}$ is planned before GLAST (which will most likely be operational after 2005-2006). AGILE might then be an ideal 'bridge' between old and new generation gamma-ray missions with an innovative design and efficient scientific management.

\section{REFERENCES}

1. Barbiellini G. et al., Nuclear Physics B 43, 253 (1995).

2. Barbiellini G. et al., Nucl. Instrum. ES Methods 354, 547 (1995).

3. Barbiellini G. et al., SPIE 2478, 239 (1995).

4. Tavani M., et al. , Mem.S.A.It. 70, 201 (1999)

5. Mereghetti S., et al. Mem.S.A.It. 70, 229 (1999) 